\title{
Micro-ribonucleic acids: potential noninvasive biomarkers for hepatocellular carcinoma
}

\author{
This article was published in the following Dove Press journal: \\ Journal of Hepatocellular Carcinoma \\ 2I May 2014 \\ Number of times this article has been viewed
}

\section{Limin $\mathrm{Li}$ \\ Wenli Diao \\ Ke Zen}

Jiangsu Engineering Research Center for MicroRNA Biology and Biotechnology, State Key Laboratory of Pharmaceutical Biotechnology, School of Life Sciences, Nanjing University, Nanjing, People's Republic of China

Correspondence: Ke Zen Jiangsu Engineering Research Center for MicroRNA Biology and Biotechnology, State Key Laboratory of Pharmaceutical Biotechnology, School of Life Sciences, Nanjing University, 22 Hankou Road, Nanjing, Jiangsu 210093,

People's Republic of China Email kzen@nju.edu.cn
Abstract: Hepatocellular carcinoma (HCC) is one of the most common malignancies globally. Each year, more than 500,000 people worldwide are diagnosed with HCC. The onset of HCC is typically unnoticeable, and the prognosis is usually poor. The early diagnosis of HCC and dynamic monitoring of this disease can contribute to more effective therapeutic interventions and improve patient outcomes. To achieve early diagnosis, more sensitive, specific, and easily detectable biomarkers are necessary. Recently, scientists have focused on identifying novel, sensitive, and minimally invasive or noninvasive biomarkers. Micro-ribonucleic acids (miRNAs) are a class of endogenous noncoding single-stranded RNAs that regulate gene expression at the posttranscriptional level. By negatively regulating target-gene expression, miRNAs play a critical role in diverse biological processes, including apoptosis, proliferation, differentiation, and developmental timing. Unique changes in miRNA expression in serum or plasma samples from HCC patients have been reported, suggesting that miRNAs may serve as novel noninvasive biomarkers for diagnosing $\mathrm{HCC}$ and evaluating therapeutic responses or as potential therapeutic targets in HCC. This review focuses on recent progress in understanding the role of miRNAs in HCC pathogenesis and progression, and highlights their diagnostic and prognostic value for HCC patients.

Keywords: hepatocellular carcinoma, miRNA, biomarker, diagnosis, prognosis

\section{Hepatocellular carcinoma risk factors, pathogenesis, diagnosis, and treatment options}

Hepatocellular carcinoma (HCC) is one of the most common malignancies worldwide, and is the third-leading cause of cancer-related death. ${ }^{1}$ The incidence of $\mathrm{HCC}$ in various regions differs significantly. ${ }^{2}$ Developing countries, including East and Southeast Asia and Central and West Africa, have the highest prevalence of HCC, due to the higher prevalence of hepatitis B virus (HBV) infection. Half of all HCC cases and deaths occur in the People's Republic of China (PRC), because HBV infection is widespread in the PRC. However, the incidence of HCC has also been increasing in developed countries, such as the $\mathrm{US}$, as a result of hepatitis $\mathrm{C}$ virus (HCV) infection and nonalcoholic steatohepatitis. ${ }^{3}$ Similar to other malignant cancers, HCC develops in response to genetic and epigenetic changes that enable cells to replicate faster or to avoid apoptosis. ${ }^{4}$ These cellular changes contribute to the high mortality rate. Many factors, including cirrhosis, ${ }^{5} \mathrm{HBV}$ infection, ${ }^{5} \mathrm{HCV}$ infection, ${ }^{6}$ alcoholism, ${ }^{7}$ smoking, ${ }^{8}$ aflatoxin, ${ }^{9}$ type 2 diabetes, ${ }^{10}$ and nonalcoholic steatohepatitis, ${ }^{11}$ can lead to HCC. In total, $70 \%-90 \%$ of HCC cases develop from chronic liver inflammation and cirrhosis. ${ }^{4}$ Cirrhosis is the most important risk factor, and is commonly caused by alcoholism, chronic HBV, or 
chronic HCV. The risk factors vary significantly in different countries. In countries where hepatitis B infection is endemic, such as in the PRC, hepatitis B is the major cause of HCC, ${ }^{2}$ whereas in countries such as the US, the major cause of HCC is cirrhosis. ${ }^{3}$ However, the mechanisms underlying the pathogenesis of HCC are still largely unknown.

It is important to monitor patients at high risk for developing HCC to diagnosis the disease at early stages. Patients with $\mathrm{HBV}$ infection, $\mathrm{HCV}$ infection and/or cirrhosis need to be closely observed for any symptoms of HCC. ${ }^{12}$ There are various risk factors for $\mathrm{HCC}$ and methods for estimating its occurrence, such as ultrasonography and measuring levels of $\alpha$-fetoprotein (AFP), des- $\gamma$-carboxy prothrombin (DCP), fucosylated fraction of AFP (AFP-L3), osteopontin, and glypican 3. Ultrasonography is the primary means of diagnosing HCC early, and is a convenient, safe, and noninvasive method. However, ultrasonography has a disadvantage, because the diagnostic results are largely dependent on the operator and equipment. Small HCC lesions can be missed in ultrasonography with background cirrhosis, even by an experienced operator. Therefore, the false-positive and -negative rates with this method are high. ${ }^{2}$ AFP is another commonly used diagnostic marker for HCC. However, it is reported that $80 \%$ of patients with small HCC lesions do not exhibit AFP upregulation. ${ }^{13}$ Combination of AFP and ultrasonography is the most commonly used diagnostic marker. Okuda et $\mathrm{al}^{14}$ reported that the sensitivity of DCP was only $35.0 \%$ and $39.3 \%$ in tumors smaller than 2 and $3 \mathrm{~cm}$, respectively. They suggested that DCP should be used in combination with AFP. AFP-L3 was reported to be a more specific marker for diagnosing HCC than AFP..$^{15}$ However, AFP-L3 is not stable, and is expressed at very low levels.

Current HCC treatment options basically follow the principles of staging-guided treatment. There are numerous staging systems for HCC. ${ }^{16}$ Barcelona Clinic Liver Cancer staging is considered the standard method for assessing the prognosis of HCC patients. This staging method is based on patient performance status. ${ }^{17}$ The recommended treatment options for and the corresponding outcomes of HCC are summarized in Table 1. For patients with very early stage HCC presenting with a single tumor that is less than $2 \mathrm{~cm}$ in diameter, ${ }^{18} 90 \%$ survival can be achieved with surgical resection. ${ }^{19}$ For patients with early stage HCC presenting with a single tumor and without portal hypertension, surgical resection should be implemented. In other cases, liver transplantation is a good choice. ${ }^{18}$ If a donor is unavailable, radiofrequency ablation is the next-best option. ${ }^{18}$ For patients with intermediate-stage $\mathrm{HCC}$, the best choice is transarterial chemoembolization, which achieves a survival time of approximately 20 months and a 3-year survival rate of $10 \%-40 \%{ }^{20,21}$ For patients with advanced disease with mild HCC-related symptoms, portal invasion, and extrahepatic metastasis, the preferred treatment method is chemotherapy, such as with sorafenib, ${ }^{22}$ which provides a survival time of approximately 11 months. ${ }^{22}$ For patients with end-stage disease, all the aforementioned treatments are ineffective. The 1 -year survival rate of these patients is less than $10 \%$, and the survival time is approximately 3 months. ${ }^{12}$

$\mathrm{HCC}$ is a rapidly progressing malignancy, and therefore most patients $(>80 \%)$ are diagnosed at the later stages, at which point surgical resection is not appropriate. Even with surgical resection, the recurrence rate is extremely high. Therefore, early surveillance and early diagnosis in high-risk populations are important, and can enable surgical treatments and improve survival. To increase the detection rate of small HCC lesions, it would be better to combine various markers, but this will increase the cost and difficulty. ${ }^{16,23}$ Although many markers

Table I Treatment options and outcomes of HCC

\begin{tabular}{|c|c|c|c|c|c|}
\hline BCLC stage ${ }^{17}$ & Tumor stage & $\begin{array}{l}\text { Child-Pugh } \\
\text { class }^{17}\end{array}$ & PS & Treatment options & Outcomes/prognosis \\
\hline Very early stage $(0)$ & $\begin{array}{l}\text { Single tumor }<2 \mathrm{~cm}, \\
\text { carcinoma in situ }\end{array}$ & A & 0 & Surgical resection & $\begin{array}{l}\text { Potentially curative treatments } \\
\text { (curative treatments } 30 \%, 5 \text {-year }\end{array}$ \\
\hline Early stage (A) & $\begin{array}{l}\text { Single tumor }<5 \mathrm{~cm} \text { or } \\
\text { three nodules }<3 \mathrm{~cm}\end{array}$ & $A$ or $B$ & 0 & $\begin{array}{l}\text { Transplantation } \\
\text { RFA } \\
\text { Surgical resection }\end{array}$ & survival $\sim 50 \%-70 \%)$ \\
\hline Intermediate stage (B) & Multinodular & $A$ or $B$ & 0 & TACE & $\begin{array}{l}\text { Palliative treatments (survival } \\
\sim 20 \text { months) } \\
\text { 3-year survival: } 10 \%-40 \%\end{array}$ \\
\hline Advanced (C) & Portal invasion & $A$ or $B$ & $0-2$ & Sorafenib & $\begin{array}{l}\text { Palliative treatments (survival } \\
\sim \text { II months) }\end{array}$ \\
\hline End stage (D) & $\begin{array}{l}\text { Liver failure, tumor } \\
\text { metastasis }\end{array}$ & C & $>2$ & Symptomatic therapy & $\begin{array}{l}\text { I-year survival rate }<10 \% \text {, } \\
\text { survival } ~ 3 \text { months }\end{array}$ \\
\hline
\end{tabular}

Abbreviations: HCC, hepatocellular carcinoma; BCLC, Barcelona Clinic Liver Cancer; PS, performance status; RFA, radiofrequency ablation; TACE, transarterial chemoembolization. 
exist, there is no single biomarker that can diagnose early stage HCC. These biomarkers usually require invasive detection methods. Therefore, to contribute to early diagnosis, treatment, and prognosis of HCC, further studies on these biomarkers and the development of novel biomarkers are urgently needed.

\section{miRNAs as posttranscriptional gene regulators}

Micro-ribonucleic acids (miRNAs) are a novel class of noncoding single-stranded RNAs that are 19-23 nucleotides in length. They are highly evolutionarily conserved, and regulate approximately $30 \%$ of all gene expression at the posttranscriptional level. ${ }^{24}$ miRNAs were first discovered in 1993; Lee et $\mathrm{al}^{25}$ discovered that lin- 4 controlled the timing of sexual development in Caenorhabditis elegans. Currently, there are
1,872 entries representing hairpin precursor miRNAs, corresponding to 2,578 mature, expressed miRNAs in Homo sapiens (miRBase release 20, http://www.mirbase.org). Functionally, miRNAs silence gene expression through two mechanisms: messenger RNA (mRNA) degradation or translational repression. If an miRNA binds to the three' untranslated region (3'UTR) of an mRNA with perfect complementarity, the miRNA will specifically direct mRNA cleavage. If an miRNA and the 3'UTR of an mRNA are partially complementary, the miRNA will inhibit translation. In animals, miRNAs are partially complementary to the 3'UTR of target mRNAs. ${ }^{26,27}$ However, the mechanism of action of miRNAs in plants is different: they are typically fully complementary, and thus result in target-gene degradation. ${ }^{24,27}$ The biogenesis of miRNAs is summarized in Figure 1.

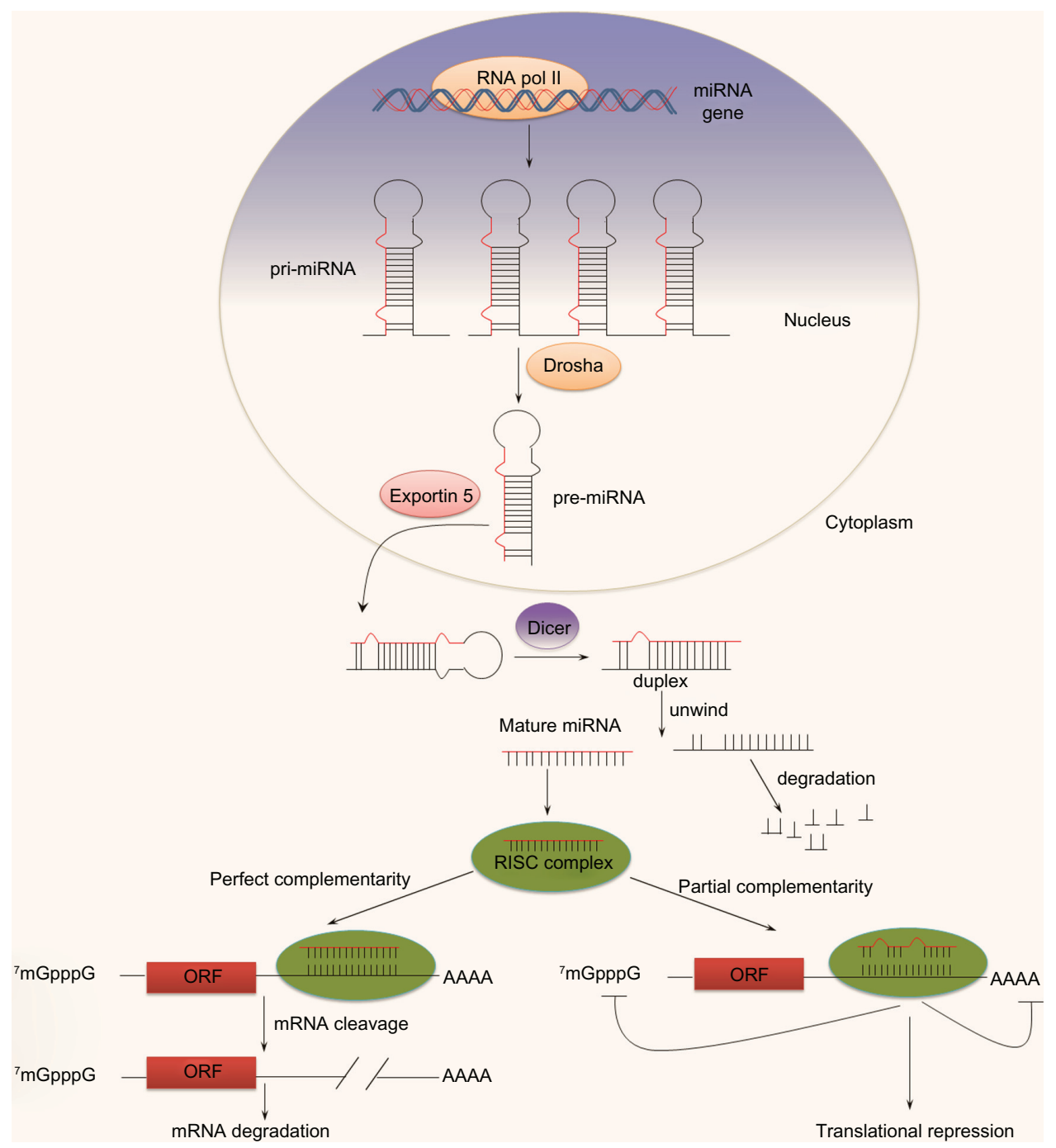

Figure I The biogenesis of micro-ribonucleic acids (miRNAs). Primary miRNAs (pri-miRNAs) are transcribed from miRNAs encoding genes by polymerase (pol) II and processed into precursor miRNA (pre-miRNA) by Drosha in the nucleus. Pre-miRNAs are next exported to the cytoplasm with the help of exportin 5 and processed into mature form by Dicer. miRNAs in cytoplasm can bind to complementary sequences on target messenger RNAs to repress translation or trigger messenger RNA cleavage. Abbreviations: ORF, open-reading frame; mRNA, messenger RNA; RISC, RNA-induced silencing complex. 
Similar to other molecules involved in regulating gene expression, the expression levels of miRNAs differ significantly in various tissues and at distinct developmental stages. ${ }^{24,26-28}$ Accumulating evidence suggests that miRNAs play an essential role in regulating gene expression at the posttranscriptional level. ${ }^{24}$ The diversity in miRNA sequence, structure, abundance, and expression make them powerful regulators of mRNA, which is involved in development, proliferation, differentiation, apoptosis, energy metabolism, and other physiological processes.$^{28}$ Therefore, alterations in miRNA expression may reflect a change in the physiological state.

\section{The role of miRNA in HCC carcinogenesis}

Abnormal miRNA expression is involved in the development of many diseases, such as cancer, ${ }^{29}$ diabetes,${ }^{30}$ inflammation, ${ }^{31}$ infection, ${ }^{31}$ cardiovascular disease, ${ }^{32}$ genetic disease, ${ }^{33}$ and nervous system disease. ${ }^{34}$ miRNAs are involved in various processes during tumor development, and have been associated with numerous cancers. ${ }^{35}$ Specific miRNA-expression profiles have been observed in cancer patients. Detecting changes in miRNA expression may become an important aspect in the early diagnosis, prognosis, and targeted treatment of cancer.

The role of miRNA in tumorigenesis is primarily focused on three aspects: miRNAs as oncogenes (oncomirs), ${ }^{36,37}$ miRNAs as tumor-suppressor genes, ${ }^{36,37}$ and miRNAs encoded by oncogenic viruses that are involved in tumorigenesis. ${ }^{38}$ The early evidence for the involvement of miRNAs in tumorigenesis was reported by Calin et al. ${ }^{39}$ They found that miR-15a and miR-16, localized at 13q14, were absent or downregulated in approximately $50 \%$ of patients with chronic lymphocytic leukemia. Cimmino et $\mathrm{al}^{40}$ determined that miR-15a and miR-16 act as tumor-suppressor genes in chronic lymphocytic leukemia by targeting B-cell CLL/lymphoma 2 (BCL2). The miR-17-92 polycistron (also known as oncomir 1), which is located at $13 \mathrm{q} 31$, is a typical example of an oncomir. Genes in this chromosomal region are involved in numerous types of tumorigenesis, such as diffuse B-cell lymphoma ${ }^{41}$ and lung cancer. ${ }^{42}$ Recent studies have shown that certain viruses encode their own miRNAs via the infected host cell. For example, Epstein-Barr virus infection is responsible for multiple types of malignancies. Infected cells express high levels of Epstein-Barr virus miRNAs, and have consistently decreased expression of cellular tumor-suppressor miRNAs. ${ }^{38}$

The development of HCC is closely related to uncontrolled cell-cycle progression and the abnormal expression of oncogenes and tumor suppressors. ${ }^{43}$ miRNAs influence HCC carcinogenesis by regulating the expression of these genes. Murakami et $\mathrm{a}^{44}$ were the first to report different miRNAexpression profiles in HCC, adjacent nontumorous tissue, and chronic hepatitis specimens using miRNA microarray. Commonly deregulated miRNAs in HCC are discussed in detail in the following sections.

\section{miR- 122}

As an abundant liver-specific miRNA, miR-122 has attracted a lot of researchers' attention. It was reported to be a typical miRNA downregulated in the liver of HCC patients. Observation of different miRNA-expression profiles in rats with HCC revealed that the let-7a, miR-21, miR-23, miR-130, miR-190, and miR-17-92 gene families were upregulated, whereas miR-122 was downregulated in rat tumors and primary human HCC tumors compared with pair-matched control..$^{45}$ In addition, cyclin $G_{1}$ could enable miR-122 to influence p53 protein stability and transcriptional activity, decreasing the invasive capacity of HCC-derived cell lines. In patients with resected HCC lesions, higher cyclin $G_{1}$ expression was associated with reduced survival, suggesting that miR-122 may be an effective molecular target in HCC. ${ }^{46} \mathrm{In}$ 2009, Tsai et al ${ }^{47}$ reported that liver-specific miR-122 was significantly downregulated in liver tumors with intrahepatic metastasis, and that it negatively regulated tumorigenesis. Restoring miR-122 expression significantly reduced in vitro migration and invasion and in vivo tumorigenesis, angiogenesis, and intrahepatic metastasis. Furthermore, they ascertained that ADAM (a disintegrin and metalloproteinase)-17 was one of the miR-122 targets involved in metastasis. When miR-122 was knocked out in mice, the mice were viable but developed temporally controlled steatohepatitis, fibrosis, and HCC. ${ }^{48}$ This suggested the importance of miR-122 in the normal physiological functioning of the liver. Interestingly, the incidence of HCC in miR-122-knockout mice recapitulates the disease incidence in humans. Moreover, various signaling pathways were disrupted in the livers of miR-122-/- mice, closely resembling the disruptions observed in human HCC. The restoration of miR-122 diminished disease manifestation and tumor incidence in miR- $122^{-/-}$mice. These findings collectively demonstrated the critical role of miR-122 in liver homeostasis and hepatocarcinogenesis.

\section{miR-22I}

miR-221 is one of the most commonly upregulated miRNAs in human cancer. miR-221 regulates the development of HCC by targeting tumor-suppressor genes, such as $B M F,{ }^{49}$ 
CDKN1B,,$^{50,51}$ CDKN1C, ${ }^{51,52}$ PTEN,,$^{53}$ IMP $3,{ }^{53}$ and human DDIT4. ${ }^{54}$ To demonstrate that miR-221 is an oncomir, Callegari et a ${ }^{55}$ developed a transgenic mouse with miR-221 overexpression in the liver, and determined that miR-221 promoted liver tumorigenicity via its target protein-coding genes $(C D K N 1 B, C D K N 1 C$, and $B M F)$, thereby providing a valuable tool for preclinical investigations of the use of antimiRNA approaches for the treatment of HCC.

\section{miR-I7-92 and miR-2I}

The miR-17-92 polycistron consists of six miRNAs: miR-17-5p, miR-18a, miR-19a, miR-19b, miR-20a, and miR-92a-1. It is reported that miR-17-92 had a negative feedback effect on suppressing HBV replication. HBV replication increases miR-17-5p, miR-20a, and miR-92a-1 expression. On the other hand, miR-20a and miR-92a-1 targeted HBV transcripts. ${ }^{56}$ Connolly et al ${ }^{57}$ established that the miR-17-92 polycistron and miR-21 were upregulated in all primary human and woodchuck HCCs. In human and woodchuck HCC cell lines, depleting tumor cells of miR-17-92 polycistron or miR-21 by specific antisense oligonucleotides resulted in a 50\% reduction in hepatocyte proliferation and growth, suggesting that miR-17-92 and miR-21 promote $\mathrm{HCC}$ tumorigenesis. The typical function of miR-21 is to promote tumor proliferation and migration by targeting phosphatase and tension homologue deleted on chromosome ten $(\mathrm{PTEN})^{58}$ and programmed cell death protein 4 (PDCD4). ${ }^{59}$ It was found that up-regulated miR-21 could strongly inhibit the protein expression of PTEN in HCC cells. ${ }^{60}$ In addition, overexpression of miR-21 suppressed and inhibition of miR-21 promoted tumor-cell proliferation, invasion, and migration by targeting PDCD4, an upstream regulator that activates transforming growth factor- $\beta{ }^{61}$

\section{$\mathrm{miR}-26 \mathrm{a}$}

miR-26a is widely expressed in various tissues. However, it was reported that miR-26a expression is downregulated in HCC cells. ${ }^{62}$ Overexpression of miR-26a in vitro induced cell-cycle arrest by targeting cyclins $\mathrm{D}_{2}$ and $\mathrm{E}_{2}$. miR-26a overexpression in a mouse model of HCC inhibited cancer cell proliferation, induced tumor-specific apoptosis, and protected the liver from disease progression. These data suggest that restoring miRNAs that are lost in diseased cells may be a general strategy for treating cancer. To restore the lost miRNA, either miRNA mimics or precursor miRNA could be used. To improve delivery efficiency, they can be chemically modified or incorporated into adenoviral or lentiviral particles. ${ }^{63}$
miR-125b

miR-125b was demonstrated to be an HCC-inhibitor gene by Lin $28 \mathrm{~B} .{ }^{64}$ It was found that miR-125b expression was downregulated in approximately $70 \%$ of HCC samples, and was negatively correlated with $\mathrm{Ki}-67$, an indicator of cell proliferation, suggesting that miR-125b inhibits the proliferation and migration of HCC cells. Furthermore, it was revealed that miR-125b suppressed cell-cycle progression by downregulating Lin28B expression, thereby upregulating p21Cip1/Waf1 expression.

\section{miR-199a/b-3p}

By performing an in-depth analysis of miRNomes, miRNAexpression profiles in human normal liver, hepatitis liver, and HCC were studied, and miRNAs were found to account for $88.2 \%$ of the miRNome in human liver. ${ }^{65}$ The three most abundantly expressed miRNAs were miR-122, miR-192, and miR-199a/b-3p, accounting for $52.0 \%, 16.9 \%$, and $4.9 \%$ of the miRNome, respectively. Subsequent analyses revealed that miR-199a/b-3p was consistently downregulated in HCC, and this decrease significantly correlated with poor survival of HCC patients. Furthermore, miR-199a/b-3p targeted the tumor-promoting protein $\mathrm{P} 21$ protein-activated kinase 4 (PAK4) to suppress HCC growth by inhibiting the PAK4/Raf/ Mitogen-activated protein kinase kinase (MEK)/extracellular signal-regulated kinase pathway both in vitro and in vivo. ${ }^{65}$ The expression of miR-199b was also investigated during the development of HCC, and was found to be involved in the progress of HCC. The expression changes of seven cancerrelated miRNAs during the early stages of HBV-related hepatocarcinogenesis in formalin-fixed paraffin-embedded dysplastic nodules, small HCCs, and corresponding nontumorous livers were evaluated, and it was demonstrated that miR-145 and miR-199b were downregulated and miR-224 upregulated in premalignant dysplastic nodules, and that these changes persisted throughout the development of HCC. ${ }^{66}$

\section{miR-I0a}

miR-10a is commonly upregulated in HCC, and is therefore another important miRNA implicated in the migration and invasion of tumor cells. miR-10a promotes epithelial-mesenchymal transition by targeting ephrin type-A receptor $4 .^{67}$

\section{miR-24, miR-I 24, and miR-629}

Hepatocyte nuclear factor (HNF)- $4 \alpha$ is an essential transcription factor for liver development and hepatocyte 
function. ${ }^{68,69}$ It was recently reported that liver tumorigenesis could be modulated by a novel feedback loop in hepatocellular carcinoma involving HNF4 $\alpha$, miR-124, interleukin 6 receptor, signal transducer and activator of transcription 3, miR-24, and miR-629. The feedback loop was governed by miR-24 and miR-629, which promote transient inhibition of $\mathrm{HNF} 4 \alpha$, resulting in miR-124 induction and signal transducer and activator of transcription 3 activation. ${ }^{70}$ These results support the use of miRNAs as potential targets for the early treatment of hepatocellular carcinoma.

\section{Tissue miRNAs as biomarkers for HCC diagnosis and prognosis}

The expression profiles of tissue miRNAs can be used as biomarkers for HCC diagnosis and prognosis. miRNAs with potential diagnostic or prognostic value for $\mathrm{HCC}$ are summarized in Table 2. miR-29 was reported to be frequently downregulated in HCC. Xiong et al reported that miR-29 regulated the apoptosis, tumorigenicity, and prognosis of HCC through targeting Bcl-2 and Mcl-1. ${ }^{71}$ miR-21, miR-31, miR-122, miR-221, and miR-222 were reported to be significantly upregulated in HCC, whereas miR-145, miR-146a, miR-200c, and miR-223 were downregulated. ${ }^{72}$ However, in intrahepatic cholangiocarcinoma samples, miR-21, miR-31, and miR-223 were upregulated, whereas miR-122, miR-145, miR-200c, miR-221, and miR-222 were downregulated. Moreover, the expression of miR-21, miR-31, miR-122, and miR-221 in HCC correlated with cirrhosis, but miR-21 and miR-221 expression was associated with tumor stage and poor prognosis. miR-203 and
miR-155 were also related to prognosis after transplantation to treat HCC. Patients with elevated miR-203 expression had significantly better recurrence-free survival and overall survival. A multivariate analysis revealed that high miR-203 expression was an independent predictor of good prognosis. ${ }^{73}$ However, patients with higher miR-155 expression had significantly worse recurrence-free survival and overall survival. Furthermore, high miR-155 expression was an independent predictor of poor prognosis and correlated with HCC invasiveness. ${ }^{74}$ miR-185 was reported to be associated with venous HCC metastasis. ${ }^{75}$ miR-185 also has clinicopathological significance and prognostic value for early stage $\mathrm{HCC}$.

miR-185 expression was investigated in a cohort of patients with early stage $\mathrm{HCC}$ who were divided into a treated recurrence group and a non-treated recurrence group, and it was discovered that low miR-185 expression correlated with increased tumor recurrence and that high miR-185 expression correlated with a lower recurrence rate. ${ }^{76}$ However, there was no direct correlation between miR-185 and clinicopathological features (ie, age, sex, alanine aminotransferase, AFP, liver cirrhosis, tumor size, tumor encapsulation, or tumor differentiation). ${ }^{76}$ Yang et al ${ }^{77}$ compared the miRNA-expression profiles in normal, chronic HBV hepatitis, HBV-positive cirrhotic, HBV-positive HCC, and corresponding normal paratumorous livers and hepatoma cells, and determined that miR-602 plays an oncogenic role in HBV-related hepatocarcinogenesis by targeting Ras association domain-containing protein 1 . These results suggested that miR-602 might be an early diagnostic marker for HBV-mediated HCC.

Table 2 Tissue micro-ribonucleic acids (miRNAs) as biomarkers for HCC diagnosis and prognosis

\begin{tabular}{|c|c|c|c|c|}
\hline miRNAs & Alteration & Diseases & Clinical significance & Reference(s) \\
\hline miR-2I, miR-223 & Up & ICC & Poor prognosis & 72 \\
\hline miR-22I & Down & & Cirrhosis related & \\
\hline miR-203 & Low & $\begin{array}{l}\text { Post-LT HCC with } \\
\text { recurrence }\end{array}$ & $\begin{array}{l}\text { Poor recurrence-free survival } \\
\text { and overall survival }\end{array}$ & 73 \\
\hline miR-I55 & High & $\begin{array}{l}\text { Post-LT HCC with } \\
\text { recurrence }\end{array}$ & $\begin{array}{l}\text { Poor recurrence-free survival } \\
\text { and overall survival }\end{array}$ & 74 \\
\hline miR-I85 & Low & Early stage HCC & High recurrence & 75,76 \\
\hline miR-602 & $U_{p}$ & HBV-positive HCC & Diagnostic marker & 77 \\
\hline miR-I8b & High & $\mathrm{HCC}$ after resection & Short relapse-free period & $78-81$ \\
\hline miR-20a & Low & $\begin{array}{l}\text { Post-LT HCC with } \\
\text { recurrence }\end{array}$ & $\begin{array}{l}\text { Poor recurrence-free survival } \\
\text { and overall survival }\end{array}$ & 82 \\
\hline miR-99a & Low & $\mathrm{HCC}$ & Poor prognosis & 83 \\
\hline $\begin{array}{l}\text { miR-I9a, miR-886-5p, } \\
\text { miR-I26, miR-223, } \\
\text { miR-24, miR-I47 }\end{array}$ & Up & OLT with recurrence & High recurrence & 84 \\
\hline
\end{tabular}

Abbreviations: ICC, intrahepatic cholangiocarcinoma; LT, liver transplantation; HBV, hepatitis B virus; OLT, orthotopic liver transplantation; HCC, hepatocellular carcinoma. 
miR-18b expression is an important marker of cell proliferation and cell adhesion, and is also a predictive marker of clinical outcome. ${ }^{78-80} \mathrm{~A}$ microarray analysis of the miRNA expression profiles in $110 \mathrm{HCC}$ samples (60 moderately, 30 poorly, and 20 well differentiated) revealed that the expression of 12 miRNAs varied significantly according to the degree of histological differentiation. Among them, the expression of miR-18b was significantly higher in poorly differentiated HCC than in well-differentiated HCC. Moreover, high miR-18b expression correlated with a significantly shorter relapse-free period than in HCC patients after surgical resection. ${ }^{81}$

The expression level of miR-20a was found to be lower in primary HCC than in normal liver, and was further decreased in patients with an HCC recurrence after liver transplantation compared with those without a recurrence. In addition, lower miR-20a expression was associated with decreased recurrence-free survival and overall survival, suggesting that miR-20a is an independent predictor of poor prognosis. miR20a may have potential as a therapeutic target and biomarker in HCC.$^{82}$ miR-99a expression is frequently downregulated in HCC tissues, implying that miR-99a may be correlated with HCC prognosis, and thus has potential as a prospective prognosis predictor. Li et al ${ }^{83}$ also reported a correlation between lower miR-99a expression and outcome for HCC patients, supporting the potential value of miR-99a as a prognostic predictor in HCC. Kaplan-Meier and Cox proportional regression analyses showed that lower miR-99a expression in HCC significantly correlated with shorter survival for HCC patients. Using multivariate analysis, miR-99a was also identified as an independent predictor for the prognosis of HCC patients.

In a study of recurrence- and prognosis-related miRNAs in HCC after orthotopic liver transplantation, miR-19a,
miR-886-5p, miR-126, miR-223, miR-24, and miR-147 were found to be upregulated in primary HCC samples from patients with recurrent HCC ${ }^{84}$ The receiver-operating characteristic (ROC) curve, Kaplan-Meier and Cox proportional regression analysis indicated that a profile of the six miRNAs might be a sensitive, specific, and independent biomarker for predicting HCC recurrence and survival.

\section{Serum miRNAs as biomarkers for HCC diagnosis and prognosis}

Recent studies have shown that there are a large number of circulating miRNAs, and that altered miRNA expression profiles are closely related to disease.$^{85}$ Circulating miRNAs were first reported in 2008. ${ }^{86,87}$ These studies demonstrated that miRNAs were present in the serum and plasma of humans and other animals, such as mice, rats, bovine fetuses, calves, and horses. Serum miRNAs are very stable, resistant to digestive enzymes, acids and bases, and less subject to temperature changes and incubation/storage times, suggesting that serum miRNAs can serve as potential biomarkers for the detection of various cancers and other diseases. ${ }^{88}$ As potential novel biomarkers, circulating miRNAs have advantages over tissue miRNAs: they are easily obtained by fast and noninvasive methods and can be quantified with high accuracy, etc. ${ }^{86,88,89}$ Lawrie et $\mathrm{a}{ }^{90}$ were the first to report that circulating miR-21 had potential as a tumor biomarker. Since then, numerous circulating miRNAs with potential as biomarkers have been reported, such as the report by $\mathrm{Hu}$ et a ${ }^{19}$ that identified four miRNAs (miR-486, miR-30d, miR-1, miR-499) that were closely related to overall survival from lung cancer. The use of circulating miRNAs as biomarkers to diagnose $\mathrm{HCC}$ has been intensely investigated. Certain miRNAs with potential diagnostic value in HCC are summarized in Table 3.

Table 3 Serum micro-ribonucleic acids (miRNAs) as biomarkers for HCC diagnosis and prognosis

\begin{tabular}{|c|c|c|c|c|}
\hline miRNAs & Alteration & Diseases & Clinical significance & Reference(s) \\
\hline miR-500, miR-92a, miR-25, miR-375, let-7f & Up & HCC, HBV-positive HCC & Diagnostic marker & $92,94,107,108$ \\
\hline miR-I8a & Up & HBV-positive HCC & Diagnostic marker & 93 \\
\hline miR-I0I & Up & HBV-positive HCC & Diagnostic marker & 94 \\
\hline miR-I7-5p, cmiR-22I & High & Post-LT HCC with recurrence & Poor overall survival & 95,96 \\
\hline miR-222 & High & HBV-positive HCC & Poor overall survival & 97 \\
\hline miR-192, miR-26a, miR-27a, miR-80I & Up & HBV-positive HCC & Diagnostic marker & 98,99 \\
\hline $\operatorname{miR}-223$ & Up & HBV-positive HCC & Diagnostic marker & $98,99,107$ \\
\hline miR-I22 & Up & HBV-positive HCC & Diagnostic marker & $98,99,103,107$ \\
\hline miR-2I & Up & HBV-positive HCC & Diagnostic marker & $98-101$ \\
\hline miR-I5b, miR-I30b & Up & $\mathrm{HCC}$ & Diagnostic marker & 101 \\
\hline miR-16 & Down & $\mathrm{HCC}$ & Diagnostic marker & 102 \\
\hline miR-885-5p & Up & HBV-positive HCC & Diagnostic marker & 106 \\
\hline miR-I & Up & Cirrhosis, HCC & Diagnostic marker & 103 \\
\hline
\end{tabular}

Abbreviations: HBV, hepatitis B virus (chronic); LT, liver transplantation; HCC, hepatocellular carcinoma. 
Circulating miR-500 was first reported with the potential to be used as a noninvasive diagnostic marker in HCC. ${ }^{92}$ As a carcinoembryonic miRNA, miR-500 was highly expressed in many human HCC cell lines and tissues. Serum miR-18a ${ }^{93}$ and miR- $101^{94}$ were also reported to have potential as noninvasive biomarkers for HBV-related HCC screening. Serum miR-18a was evaluated in $101 \mathrm{HBV}$ patients with $\mathrm{HCC}, 30$ patients with HBV alone, and 30 with cirrhosis, as well as 60 healthy patients. miR-101 expression was investigated in tissue and serum from patients with HBV-related HCC and from healthy controls. The data demonstrated that miR-101 was downregulated in HBV-related HCC tissue compared with adjacent noncancerous and normal tissue. Importantly, serum miR-101 levels were significantly higher in HBV-related HCC patients than in normal controls, and the increase correlated with HBV surface-antigen positivity, HBV deoxyribonucleic acid levels, and tumor size. These studies further confirmed that plasma miRNA panels have considerable clinical value for the diagnosis of early stage HCC.

Cancer tissues, including those from HCC, typically contain upregulated miR-17-5p, miR-221, and miR-222. Serum levels of miR-17-5p, miR-221, and miR-222 are associated with the development of HCC, and could be noninvasive biomarkers to predict prognosis in HCC patients. miR-17-5p serum levels were significantly downregulated in an HCC postoperative group, but were upregulated in an HCC relapsed group, and high serum levels of miR-17-5p correlated with reduced overall survival; ${ }^{95}$ miR-221 serum levels were correlated with clinicopathologic features of $\mathrm{HCC}$, and prognosis and the overall survival rate of HCC patients with high serum levels of miR-221 were significantly lower than that of patients with the low serum levels of miR-221;96 miR-222 serum levels were also significantly correlated with cirrhosis, tumor number, portal vein tumor thrombosis, and Classification of Malignant Tumors (TNM) stage, but were not correlated with serum AFP levels, tumor size, or surface antigen of the hepatitis B virus (HbsAg) ${ }^{97}$ The median overall survival in the miR-222 high-expression group was also significantly shorter than that in the low miR222 expression group.

miR-21, miR-223, and miR-122 are commonly deregulated miRNAs in HCC. By investigating miRNA expression in plasma from healthy controls, chronic HBV (CHB), cirrhosis, and HBV-related HCC, Zhou et $\mathrm{a}^{98}$ found a profile of seven miRNAs consisting of miR-122, miR-192, miR-21, miR-223, miR-26a, miR-27a, and miR-801 could differentiate HCC from healthy CHB or cirrhosis with high accuracy. $\mathrm{Xu}$ et $\mathrm{al}^{99}$ revealed that miR-21, miR-122, and
miR-223 could discriminate HBV-related HCC or CHB from healthy controls, but could not discriminate HBV-related HCC from CHB. They found that serum levels of miR-21, miR-122, and miR-223 were upregulated in HBV-related HCC patients compared with healthy controls, and miR-21 and miR-122 were higher in patients with CHB than in HCC patients. Serum miR-21 levels also correlated with clinical parameters in patients with different stages of chronic $\mathrm{HCV}$ (CHC) infection and CHC-related HCC. ${ }^{100}$ It was ascertained that miR-21 levels were higher in patients with $\mathrm{CHC}$ than in healthy controls, but the serum miR-21 levels could not discriminate $\mathrm{CHC}$ from CHC-related HCC. These results suggest that miR-21 may be a potential biomarker for distinguishing $\mathrm{CHB}, \mathrm{CHC}, \mathrm{CHB}-$ related $\mathrm{HCC}$, or CHC-related HCC from healthy controls, but not be a good biomarker for distinguishing $\mathrm{CHB}$ or $\mathrm{CHC}$ from CHB-related $\mathrm{HCC}$ or CHC-related HCC.

By studying 96 patients with $\mathrm{HCC}$ who received primary curative surgical resection, 57 patients with HCC, and 30 healthy controls, Liu et a ${ }^{101}$ demonstrated that the combination of miR-15b and miR-130b had potential value for HCC screening in patients from the PRC. miR-15b, miR-21, miR-130b, and miR-183 were highly expressed in tumors and serum, but were markedly reduced after surgery. The accuracy of combined miR-15b and miR-130b is better than AFP in predicting early stage and low-AFP HCC cases. Qu et $\mathrm{al}^{102}$ quantitated the serum levels of miR-16, miR-195, and miR-199a in 105 patients with HCC, 107 patients with chronic liver disease, and 71 normal controls, and ascertained that miR-16 and miR-199a serum levels were significantly reduced in HCC patients. The sensitivity and specificity of miR-16 alone or in combination with other markers (AFP, AFP-L3, and DCP) in diagnosing HCC were $92.4 \%$ and $78.5 \%$, respectively. Köberle et a $1^{103}$ reported that serum miR-122 and miR-1 levels have prognostic potential in HCC. In 195 HCC patients and 54 patients with liver cirrhosis, miR-1 and miR-122 levels correlated with overall survival, Cancer of the Liver Italian Program score, Barcelona Clinic Liver Cancer stage, ${ }^{104,105}$ clinical chemistry parameters, and tumor-specific treatment, indicating that serum miR-1 is a novel independent biomarker of overall survival in HCC patients, and may therefore improve the predictive value of classical HCC staging scores.

Serum miR-885-5p could also be a biomarker for HCC. ${ }^{106}$ miR-885-5p, miR-574-3p, miR-224, miR-215, and miR-146a were reported to be upregulated in HCC and liver cancer. But only the serum levels of miR-885-5p were significantly higher in patients with $\mathrm{CHB}$, cirrhosis, and HCC compared 
with normal controls and patients with colorectal cancer. ${ }^{106}$ Li et $\mathrm{al}^{107}$ employed a strategy of initial screening by Solexa sequencing (Illumina, San Diego, CA, USA) followed by validation with $\operatorname{TaqMan}^{\circledR}$ (Life Technologies, Carlsbad, CA, USA) probe-based quantitative reverse-transcription polymerase chain reaction to ascertain the miRNA profiles of 513 subjects ( 210 controls and $135 \mathrm{HBV}^{-}, 48 \mathrm{HCV}^{-}$, and $120 \mathrm{HCC}$-affected individuals). Solexa sequencing revealed that 13 miRNAs were upregulated in the serum of HBV patients compared with normal controls, and the combination of these 13 miRNAs accurately discriminated HBV cases from controls and $\mathrm{HCV}$ cases, and also discriminated HBVpositive $\mathrm{HCC}$ cases from control and $\mathrm{HBV}$ cases. The combination of miR-25, miR-375, and let-7f clearly separated HCC cases from controls, and miR-375 alone had an ROC curve of 0.96 (specificity 96\%, sensitivity 100\%) in predicting HCC. Their study demonstrated for the first time that serum miRNA profiles can serve as novel and noninvasive biomarkers for HBV infection and HBV-positive HCC. miR-92a was also reported to be upregulated in the plasma of patients with HCC compared with normal controls. However, compared with patients before tumor resection, it was downregulated in plasma from patients after tumor resection. ${ }^{108}$ Therefore, all of these studies suggest that altered miRNA-expression profiles are associated with aspects of $\mathrm{HCC}$, and are expected to be novel, noninvasive biomarkers for the diagnosis of different stages of HCC.

\section{Discussion and conclusion}

HCC is a highly lethal cancer that is usually diagnosed at the late stage. At diagnosis, patients have usually missed the best treatment window. The main reasons for this clinical outcome for HCC patients are the lack of reliable biomarkers for the early diagnosis of HCC and the paucity of effective treatment options to manage $\mathrm{HCC}$ patients. Identifying tumor biomarkers and developing accurate detection methods have long been the focus of cancer research, including in studies on HCC. Biomarkers play an important role in the early diagnosis, individualized treatment, prognosis, and numerous other aspects of HCC. Despite the growing number of HCC biomarkers that have been identified and used for HCC screening, diagnosis, and treatment, there are still significant deficiencies in clinical applications. In addition, current procedures for biomarker detection are complicated and invasive, which limits their clinical utility. Because of certain disadvantages, such as the variety of tumor-specific differentiation categories, low overall sensitivity, relatively high requirements for sample storage conditions, and the significant expense of detection methods, it is difficult to promote the use of existing HCC markers widely. Therefore, it is necessary to identify novel, sensitive, and convenient biomarkers for $\mathrm{HCC}$ that can remedy the disadvantages of the existing markers.

miRNAs that control the expression of numerous target genes in HCC have been well documented. As reported in many studies, certain miRNAs function as oncogenes, whereas others act as tumor suppressors. Extensive studies have demonstrated that HCC patients with different disease etiologies or at different disease stages exhibit specific miRNA profiles (either in circulation or in HCC tissue) that are associated with HCC progression or metastasis. These specific miRNAs have potential as diagnostic or prognostic HCC biomarkers. Although tissue miRNAs can be used as HCC biomarkers, surgery or invasive biopsies would still be required to obtain tissue for miRNA detection. Therefore, tissue miRNAs are not satisfactory for the diagnosis of early stage $\mathrm{HCC}$ or for applications in extensively screening for or preventing HCC. Based on this, researchers have turned their attention to circulating miRNAs, which are more readily available and can be collected during a routine physical examination.

Because blood circulates to all parts of the body and transports nutrients to and removes waste from cells, serum miRNAs reflect the physiological and pathological condition of the entire organism, and serum-test results have real significance for human health. The most probable sources of circulating miRNA involve three different pathways: 1) passive leakage from broken cells due to tissue injury, chronic inflammation, cell apoptosis, or necrosis, or from cells with a short half-life, such as platelets; ${ }^{109} 2$ ) active secretion via microvesicles, including exosomes and shedding vesicles; $;^{88,89,110,111}$ or 3 ) active secretion through a microvesicle-free, RNA-binding protein-dependent pathway. ${ }^{112,113}$ These provide the functional and applied basis of serum/plasma miRNA. Furthermore, serum-based detection is noninvasive, and thus can be applied to screening, prevention, and early diagnosis.

Compared with traditional biomarkers, serum miRNA has four main advantages: 1) miRNAs are ubiquitous in the serum/plasma of normal subjects and patients with various physiological conditions, tumor types, and disease stages; 2) serum/plasma miRNAs are stable, and the detection and quantitation methods are minimally invasive and easy to perform; ${ }^{107,114} 3$ ) the detection of serum/ plasma miRNAs is a real-time, comprehensive diagnostic method that can clearly define the progression of cancer 
and comprehensively reflect the disease status of a patient; ${ }^{88}$ and 4) the screening of a serum/plasma miRNA fingerprint requires a rigorous multistage validation and evaluation system. Solexa sequencing technology was initially used for direct sequencing of serum/plasma miRNAs to perform cancer-specific miRNA-expression profiling. Realtime fluorescence methods were subsequently applied to perform quantitative reverse-transcription polymerase chain reaction screening of miRNA-expression profiles in large samples for independent validation. Preliminary evaluations and accuracy assessments of serum/plasma miRNAs as biomarkers could be performed using statistical analysis software and epidemiological data-analysis programs. ${ }^{88,114-117}$ The application of these procedures and policies would not only ensure the accuracy of serum/ plasma miRNA biomarkers in clinical applications but also provide a method and strategy for the development of other disease biomarkers.

Although detecting the levels of circulating miRNAs for early diagnosis, individualized treatment, and prognosis of cancers have been gratifying achievements, clinical application still has a long way to go. The main issues to be addressed include: 1) looking for tumor-specific or organspecific miRNA; 2) there are individual differences in miRNA expression, and miRNA-expression profiles vary based on the etiology of HCC (viral hepatitis, cirrhosis, etc) and on the immune status of HCC patients; 3 ) validating these biomarkers in larger sample sizes and in different ethnic populations; and 4) establishing conventional detection methods that can be carried out in the clinical laboratory, including standardized detection systems, standards, reference materials, and quality-control materials.

In conclusion, the advancement of research and technology in the fields of microarrays, sequencing, quantitative techniques, and bioinformatics will enable the discovery of numerous specific miRNAs that are potential biomarkers for the early detection of or prognosis for HCC. As emerging tumor biomarkers, miRNAs have good prospects for the future diagnosis and treatment of cancer.

\section{Acknowledgments}

This work was supported by grants from the National Basic Research Program of China (973 Program, 2012CB517603, and 2011CB504803), the National Natural Science Foundation of China (31301061), the Natural Science Foundation of Jiangsu Province (BK2011013, BK20130564), and the Specialized Research Fund for the Doctoral Program of Higher Education (20130091120037).

\section{Disclosure}

The authors report no conflicts of interest in this work.

\section{References}

1. Qi J, Wang J, Katayama H, Sen S, Liu SM. Circulating microRNAs (cmiRNAs) as novel potential biomarkers for hepatocellular carcinoma. Neoplasma. 2013;60(2):135-142.

2. Kim DY, Han KH. Epidemiology and surveillance of hepatocellular carcinoma. Liver Cancer. 2012;1(1):2-14.

3. El-Serag HB. Epidemiology of hepatocellular carcinoma in USA. Hepatol Res. 2007;37 Suppl 2:S88-S94.

4. El-Serag HB, Rudolph KL. Hepatocellular carcinoma: epidemiology and molecular carcinogenesis. Gastroenterology. 2007;132(7): 2557-2576.

5. Nishioka K, Levin AG, Simons MJ. Hepatitis B antigen, antigen subtypes, and hepatitis B antibody in normal subjects and patients with liver disease. Bull World Health Organ. 1975;52(3):293-300.

6. Alter MJ. Epidemiology of hepatitis C virus infection. World $J$ Gastroenterol. 2007;13(17):2436-2441.

7. Lund B, Sorensen OH, Hilden M, Lund B. The hepatic conversion of vitamin $\mathrm{D}$ in alcoholics with varying degrees of liver affection. Acta Med Scand. 1977;202(3):221-224.

8. Austin H, Delzell E, Grufferman S, et al. A case-control study of hepatocellular carcinoma and the hepatitis B virus, cigarette smoking, and alcohol consumption. Cancer research. 1986;46(2):962-966.

9. Onyemelukwe CG, Nirodi C, West CE. Aflatoxin B1 in hepatocellular carcinoma. Trop Geogr Med. 1980;32(3):237-240.

10. Lai MS, Hsieh MS, Chiu YH, Chen TH. Type 2 diabetes and hepatocellular carcinoma: a cohort study in high prevalence area of hepatitis virus infection. Hepatology. 2006;43(6):1295-1302.

11. Zen Y, Katayanagi K, Tsuneyama K, Harada K, Araki I, Nakanuma Y. Hepatocellular carcinoma arising in non-alcoholic steatohepatitis. Pathol Int. 2001;51(2):127-131.

12. Bruix J, Sherman M. Management of hepatocellular carcinoma. Hepatology. 2005;42(5):1208-1236.

13. Lok AS, Sterling RK, Everhart JE, et al. Des-gamma-carboxy prothrombin and alpha-fetoprotein as biomarkers for the early detection of hepatocellular carcinoma. Gastroenterology. 2010;138(2): 493-502.

14. Okuda H, Nakanishi T, Takatsu K, et al. Measurement of serum levels of des-gamma-carboxy prothrombin in patients with hepatocellular carcinoma by a revised enzyme immunoassay kit with increased sensitivity. Cancer. 1999;85(4):812-818.

15. Choi JY, Jung SW, Kim HY, et al. Diagnostic value of AFP-L3 and PIVKA-II in hepatocellular carcinoma according to total-AFP. World J Gastroenterol. 2013;19(3):339-346.

16. Yang HI, Yuen MF, Chan HLY, et al. Risk estimation for hepatocellular carcinoma in chronic hepatitis B (REACH-B): development and validation of a predictive score. Lancet Oncol. 2011;12(6): 568-574

17. Forner A, Reig ME, de Lope CR, Bruix J. Current strategy for staging and treatment: the BCLC update and future prospects. Semin Liver Dis. 2010;30(1):61-74.

18. Bruix J, Sherman M. Management of hepatocellular carcinoma: an update. Hepatology. 2011;53(3):1020-1022.

19. Roayaie S, Obeidat K, Sposito C, et al. Resection of hepatocellular cancer $\leq 2 \mathrm{~cm}$ : results from two Western centers. Hepatology. 2013;57(4):1426-1435.

20. Kudo M, Han KH, Kokudo N, et al. Liver Cancer Working Group report. Jpn J Clin Oncol. 2010;40 Suppl 1:i19-i27.

21. Vitale A, Saracino E, Boccagni P, et al. Validation of the BCLC prognostic system in surgical hepatocellular cancer patients. Transplant Proc. 2009;41(4):1260-1263.

22. Llovet JM, Ricci S, Mazzaferro V, et al. Sorafenib in advanced hepatocellular carcinoma. $N$ Engl J Med. 2008;359(4):378-390. 
23. Kurosaki M, Hiramatsu N, Sakamoto M, et al. Data mining model using simple and readily available factors could identify patients at high risk for hepatocellular carcinoma in chronic hepatitis C. J Hepatol. 2012;56(3):602-608.

24. Bartel DP. MicroRNAs: genomics, biogenesis, mechanism, and function. Cell. 2004;116(2):281-297.

25. Lee RC, Feinbaum RL, Ambros V. The C. elegans heterochronic gene lin-4 encodes small RNAs with antisense complementarity to lin-14. Cell. 1993;75(5):843-854.

26. Ambros V. The functions of animal microRNAs. Nature. 2004; 431(7006):350-355.

27. Bartel DP. MicroRNAs: target recognition and regulatory functions. Cell. 2009;136(2):215-233.

28. Ambros V. MicroRNA pathways in flies and worms: growth, death, fat, stress, and timing. Cell. 2003;113(6):673-676.

29. McManus MT. MicroRNAs and cancer. Semin Cancer Biol. 2003; 13(4):253-258.

30. Poy MN, Eliasson L, Krutzfeldt J, et al. A pancreatic islet-specific microRNA regulates insulin secretion. Nature. 2004;432(7014): 226-230.

31. Chen J, Li WX, Xie D, Peng JR, Ding SW. Viral virulence protein suppresses RNA silencing-mediated defense but upregulates the role of microrna in host gene expression. Plant Cell. 2004;16(5): 1302-1313.

32. Yang B, Lin H, Xiao J, et al. The muscle-specific microRNA miR-1 regulates cardiac arrhythmogenic potential by targeting GJA1 and KCNJ2. Nat Med. 2007;13(4):486-491.

33. Paulson H. RNA interference as potential therapy for neurodegenerative disease: applications to inclusion-body myositis? Neurology. 2006; 66(2 Suppl 1):S114-S117.

34. Davidson BL, Boudreau RL. RNA interference: a tool for querying nervous system function and an emerging therapy. Neuron. 2007;53(6): 781-788.

35. Lu J, Getz G, Miska EA, et al. MicroRNA expression profiles classify human cancers. Nature. 2005;435(7043):834-838.

36. Hammond SM. RNAi, microRNAs, and human disease. Cancer Chemother Pharmacol. 2006;58 Suppl 1:S63-S68.

37. Schmid CA, Craig VJ, Müller A, Flori M. The role of microRNAs in the pathogenesis and treatment of hematopoietic malignancies. Curr Pharm Des. 2013;19(7):1201-1210.

38. Marquitz AR, Mathur A, Chugh PE, Dittmer DP, Raab-Traub N. Expression profile of microRNAs in Epstein-Barr virus-infected AGS gastric carcinoma cells. J Virol. 2014;88(2):1389-1393.

39. Calin GA, Dumitru CD, Shimizu M, et al. Frequent deletions and down-regulation of micro-RNA genes miR15 and miR16 at 13q14 in chronic lymphocytic leukemia. Proc Natl Acad Sci USA. 2002;99(24): 15524-15529.

40. Cimmino A, Calin GA, Fabbri M, et al. miR-15 and miR-16 induce apoptosis by targeting BCL2. Proc Natl Acad Sci U S A. 2005;102(39): 13944-13949.

41. He L, Thomson JM, Hemann MT, et al. A microRNA polycistron as a potential human oncogene. Nature. 2005;435(7043):828-833.

42. Hayashita Y, Osada H, Tatematsu Y, et al. A polycistronic microRNA cluster, miR-17-92, is overexpressed in human lung cancers and enhances cell proliferation. Cancer Res. 2005;65(21):9628-9632.

43. Sun W, Zhong F, Zhi L, Zhou G, He F. Systematic -omics analysis of HBV-associated liver diseases. Cancer Lett. 2009;286(1):89-95.

44. Murakami Y, Yasuda T, Saigo K, et al. Comprehensive analysis of microRNA expression patterns in hepatocellular carcinoma and non-tumorous tissues. Oncogene. 2006;25(17):2537-2545.

45. Kutay H, Bai S, Datta J, et al. Downregulation of miR-122 in the rodent and human hepatocellular carcinomas. $J$ Cell Biochem. 2006;99(3):671-678.

46. Fornari F, Gramantieri L, Giovannini C, et al. MiR-122/cyclin G1 interaction modulates p53 activity and affects doxorubicin sensitivity of human hepatocarcinoma cells. Cancer Res. 2009;69(14): 5761-5767.
47. Tsai WC, Hsu PW, Lai TC, et al. MicroRNA-122, a tumor suppressor microRNA that regulates intrahepatic metastasis of hepatocellular carcinoma. Hepatology. 2009;49(5):1571-1582.

48. Tsai WC, Hsu SD, Hsu CS, et al. MicroRNA-122 plays a critical role in liver homeostasis and hepatocarcinogenesis. J Clin Invest. 2012;122(8): 2884-2897.

49. Gramantieri L, Fornari F, Ferracin M, et al. MicroRNA-221 targets Bmf in hepatocellular carcinoma and correlates with tumor multifocality. Clin Cancer Res. 2009;15(16):5073-5081.

50. Galardi S, Mercatelli N, Giorda E, et al. miR-221 and miR-222 expression affects the proliferation potential of human prostate carcinoma cell lines by targeting p27Kip1. J Biol Chem. 2007;282(32):23716-23724.

51. Medina R, Zaidi SK, Liu CG, et al. MicroRNAs 221 and 222 bypass quiescence and compromise cell survival. Cancer Res. 2008;68(8):2773-2780.

52. Fornari F, Gramantieri L, Ferracin M, et al. MiR-221 controls CDKN1C/ p57 and CDKN1B/p27 expression in human hepatocellular carcinoma. Oncogene. 2008;27(43):5651-5661.

53. Garofalo M, Di Leva G, Romano G, et al. miR-221\&222 regulate TRAIL resistance and enhance tumorigenicity through PTEN and TIMP3 downregulation. Cancer Cell. 2009;16(6):498-509.

54. Pineau P, Volinia S, McJunkin K, et al. miR-221 overexpression contributes to liver tumorigenesis. Proc Natl Acad Sci U SA. 2010;107(1): 264-269.

55. Callegari E, Elamin BK, Giannone F, et al. Liver tumorigenicity promoted by microRNA-221 in a mouse transgenic model. Hepatology. 2012;56(3):1025-1033.

56. Jung YJ, Kim JW, Park SJ, et al. c-Myc-mediated overexpression of miR-17-92 suppresses replication of hepatitis B virus in human hepatoma cells. J Med Virol. 2013;85(6):969-978.

57. Connolly E, Melegari M, Landgraf P, et al. Elevated expression of the miR-17-92 polycistron and miR-21 in hepadnavirus-associated hepatocellular carcinoma contributes to the malignant phenotype. Am J Pathol. 2008;173(3):856-864.

58. Meng FY, Henson R, Lang M, et al. Involvement of human micro-RNA in growth and response to chemotherapy in human cholangiocarcinoma cell lines. Gastroenterology. 2006;130(7):2113-2129.

59. Asangani IA, Rasheed SA, Nikolova DA, et al. MicroRNA-21 (miR-21) post-transcriptionally downregulates tumor suppressor Pdcd4 and stimulates invasion, intravasation and metastasis in colorectal cancer. Oncogene. 2008;27(15):2128-2136.

60. Meng FY, Henson R, Wehbe-Janek H, Ghoshal K, Jacob ST, Patel T. MicroRNA-21 regulates expression of the PTEN tumor suppressor gene in human hepatocellular cancer. Gastroenterology. 2007;133(2): 647-658.

61. Davis BN, Hilyard AC, Lagna G, Hata A. SMAD proteins control DROSHA-mediated microRNA maturation. Nature. 2008; 454(7200):56-61.

62. Kota J, Chivukula RR, O'Donnell KA, et al. Therapeutic microRNA delivery suppresses tumorigenesis in a murine liver cancer model. Cell. 2009;137(6):1005-1017.

63. Giordano S, Columbano A. MicroRNAs: new tools for diagnosis, prognosis, and therapy in hepatocellular carcinoma? Hepatology. 2013;57(2):840-847.

64. Liang L, Wong CM, Ying Q, et al. MicroRNA-125b suppressed human liver cancer cell proliferation and metastasis by directly targeting oncogene LIN28B2. Hepatology. 2010;52(5):1731-1740.

65. Hou J, Lin L, Zhou WP, et al. Identification of miRNomes in human liver and hepatocellular carcinoma reveals miR-199a/b-3p as therapeutic target for hepatocellular carcinoma. Cancer Cell. 2011;19(2): 232-243.

66. Gao P, Wong CC, Tung EK, Lee JM, Wong CM, Ng IO. Deregulation of microRNA expression occurs early and accumulates in early stages of HBV-associated multistep hepatocarcinogenesis. J Hepatol. 2011;54(6):1177-1184.

67. Yan Y, Luo YC, Wan HY, et al. MicroRNA-10a is involved in the metastatic process by regulating Eph tyrosine kinase receptor A4-mediated epithelial-mesenchymal transition and adhesion in hepatoma cells. Hepatology. 2013;57(2):667-677. 
68. Battle MA, Konopka G, Parviz F, et al. Hepatocyte nuclear factor 4alpha orchestrates expression of cell adhesion proteins during the epithelial transformation of the developing liver. Proc Natl Acad Sci US A. 2006;103(22):8419-8424.

69. Kamiya A, Inoue Y, Gonzalez FJ. Role of the hepatocyte nuclear factor 4alpha in control of the pregnane $\mathrm{X}$ receptor during fetal liver development. Hepatology. 2003;37(6):1375-1384.

70. Hatziapostolou M, Polytarchou C, Aggelidou E, et al. An HNF4alphamiRNA inflammatory feedback circuit regulates hepatocellular oncogenesis. Cell. 2011;147(6):1233-1247.

71. Xiong Y, Fang JH, Yun JP, et al. Effects of microRNA-29 on apoptosis, tumorigenicity, and prognosis of hepatocellular carcinoma. Hepatology. 2010;51(3):836-845.

72. Karakatsanis A, Papaconstantinou I, Gazouli M, Lyberopoulou A, Polymeneas G, Voros D. Expression of microRNAs, miR-21, miR-31, miR-122, miR-145, miR-146a, miR-200c, miR-221, miR-222, and miR-223 in patients with hepatocellular carcinoma or intrahepatic cholangiocarcinoma and its prognostic significance. Mol Carcinog. 2013;52(4):297-303.

73. Chen HY, Han ZB, Fan JW, et al. miR-203 expression predicts outcome after liver transplantation for hepatocellular carcinoma in cirrhotic liver. Med Oncol. 2012;29(3):1859-1865.

74. Han ZB, Chen HY, Fan JW, Wu JY, Tang HM, Peng ZH. Up-regulation of microRNA-155 promotes cancer cell invasion and predicts poor survival of hepatocellular carcinoma following liver transplantation. J Cancer Res Clin Oncol. 2012;138(1):153-161.

75. Zheng ZF, Su HF, Zou Y, Peng Z, Wu SX. [Expression profiles of microRNAs in radioresistant esophageal cell line]. Zhonghua Yi Xue Za Zhi. 2011;91(9):639-642. Chinese.

76. Zhi QM, Zhu JJ, Guo XB, et al. Metastasis-related miR-185 is a potential prognostic biomarker for hepatocellular carcinoma in early stage. Biomed Pharmacother. 2013;67(5):393-398.

77. Yang LA, Ma ZL, Wang DD, Zhao WY, Chen LB, Wang GB. MicroRNA-602 regulating tumor suppressive gene RASSF1A is overexpressed in hepatitis B virus-infected liver and hepatocellular carcinoma. Cancer Biol Ther. 2010;9(10):803-808.

78. Fonseca-Sanchéz MA, Pérez-Plasencia C, Fernández-Retana J, et al. MicroRNA-18b is upregulated in breast cancer and modulates genes involved in cell migration. Oncol Rep. 2013;30(5):2399-2410.

79. Jazirehi AR, Torres-Collado AX, Nazarian R. Role of miR-18b/ MDM2/p53 circuitry in melanoma progression. Epigenomics. 2013;5(3):254.

80. Yu X, Zhen Y, Yang H, et al. Loss of connective tissue growth factor as an unfavorable prognosis factor activates miR-18b by PI3K/AKT/C-Jun and C-Myc and promotes cell growth in nasopharyngeal carcinoma. Cell Death Dis. 2013;4:e634.

81. Murakami Y, Tamori A, Itami S, et al. The expression level of miR-18b in hepatocellular carcinoma is associated with the grade of malignancy and prognosis. BMC Cancer. 2013;13:99.

82. Fan MQ, Huang CB, Gu Y, Xiao Y, Sheng JX, Zhong L. Decrease expression of microRNA-20a promotes cancer cell proliferation and predicts poor survival of hepatocellular carcinoma. J Exp Clin Canc Res. 2013;32(1):21.

83. Li D, Liu XG, Lin L, et al. MicroRNA-99a inhibits hepatocellular carcinoma growth and correlates with prognosis of patients with hepatocellular carcinoma. J Biol Chem. 2011;286(42):36677-36685.

84. Han ZB, Zhong L, Teng MJ, et al. Identification of recurrence-related microRNAs in hepatocellular carcinoma following liver transplantation. Mol Oncol. 2012;6(4):445-457.

85. Berger F, Reiser MF. Micro-RNAs as potential new molecular biomarkers in oncology: have they reached relevance for the clinical imaging sciences? Theranostics. 2013;3(12):932-941.

86. Chen X, Ba Y, Ma LJ, et al. Characterization of microRNAs in serum: a novel class of biomarkers for diagnosis of cancer and other diseases. Cell Res. 2008;18(10):997-1006.

87. Mitchell PS, Parkin RK, Kroh EM, et al. Circulating microRNAs as stable blood-based markers for cancer detection. Proc Natl Acad Sci U SA. 2008;105(30):10513-10518.
88. Zen K, Zhang CY. Circulating microRNAs: a novel class of biomarkers to diagnose and monitor human cancers. Med Res Rev. 2012;32(2): 326-348.

89. Li L, Zhu D, Huang L, et al. Argonaute 2 complexes selectively protect the circulating microRNAs in cell-secreted microvesicles. PloS One. 2012;7(10):e46957.

90. Lawrie CH, Soneji S, Marafioti T, et al. MicroRNA expression distinguishes between germinal center B cell-like and activated B cell-like subtypes of diffuse large B cell lymphoma. Int J Cancer. 2007;121(5): 1156-1161.

91. Hu ZB, Chen X, Zhao Y, et al. Serum microRNA signatures identified in a genome-wide serum microRNA expression profiling predict survival of non-small-cell lung cancer. J Clin Oncol. 2010;28(10): 1721-1726.

92. Yamamoto Y, Kosaka N, Tanaka M, et al. MicroRNA-500 as a potential diagnostic marker for hepatocellular carcinoma. Biomarkers. 2009;14(7):529-538.

93. Li LH, Guo ZJ, Wang JH, Mao Y, Gao Q. Serum miR-18a: a potential marker for hepatitis B virus-related hepatocellular carcinoma screening. Digest Dis Sci. 2012;57(11):2910-2916.

94. Fu Y, Wei X, Tang C, et al. Circulating microRNA-101 as a potential biomarker for hepatitis B virus-related hepatocellular carcinoma. Oncol Lett. 2013;6(6):1811-1815.

95. Zheng J, Dong P, Gao S, Wang N, Yu F. High expression of serum miR-17-5p associated with poor prognosis in patients with hepatocellular carcinoma. Hepatogastroenterology. 2013;60(123):549-552.

96. Li JP, Wang YP, Yu WJ, Chen J, Luo JP. Expression of serum miR221 in human hepatocellular carcinoma and its prognostic significance. Biochem Biophys Res Commun. 2011;406(1):70-73.

97. Zhan MX, Li Y, Hu BS, et al. [Expression of serum microRNAs (miR-222, miR-181, miR-216) in human hepatocellular carcinoma and its clinical significance]. Zhonghua Yi Xue Za Zhi. 2013;93(23): 1830-1832. Chinese.

98. Zhou J, Yu L, Gao X, et al. Plasma microRNA panel to diagnose hepatitis B virus-related hepatocellular carcinoma. J Clin Oncol. 2011;29(36):4781-4788.

99. Xu J, Wu C, Che X, et al. Circulating microRNAs, miR-21, miR-122, and miR-223, in patients with hepatocellular carcinoma or chronic hepatitis. Mol Carcinog. 2011;50(2):136-142.

100. Bihrer V, Waidmann O, Friedrich-Rust M, et al. Serum microRNA-21 as marker for necroinflammation in hepatitis $\mathrm{C}$ patients with and without hepatocellular carcinoma. PloS One. 2011;6(10):e26971.

101. Liu AM, Yao TJ, Wang W, et al. Circulating miR-15b and miR-130b in serum as potential markers for detecting hepatocellular carcinoma: a retrospective cohort study. BMJ Open. 2012;2(2):e000825.

102. Qu KZ, Zhang K, Li HR, Afdhal NH, Albitar M. Circulating microRNAs as biomarkers for hepatocellular carcinoma. J Clin Gastroenterol. 2011;45(4):355-360.

103. Köberle V, Kronenberger B, Pleli T, et al. Serum microRNA-1 and microRNA-122 are prognostic markers in patients with hepatocellular carcinoma. Eur J Cancer. 2013;49(16):3442-3449.

104. [No authors listed]. A new prognostic system for hepatocellular carcinoma: a retrospective study of 435 patients: the Cancer of the Liver Italian Program (CLIP) investigators. Hepatology. 1998;28:751-755.

105. Llovet JM, Fuster J, Bruix J, Barcelona-Clinic Liver Cancer Group. The Barcelona approach: diagnosis, staging, and treatment of hepatocellular carcinoma. Liver Transpl. 2004;10:S115-120.

106. Gui JH, Tian YP, Wen XY, et al. Serum microRNA characterization identifies miR-885-5p as a potential marker for detecting liver pathologies. Clin Sci. 2011;120(5-6):183-193.

107. Li LM, Hu ZB, Zhou ZX, Chen X, Liu FY, Zhang JF. Serum microRNA profiles serve as novel biomarkers for HBV infection and diagnosis of HBV-positive hepatocarcinoma. Cancer Res. 2011;70(23): 9798-9807.

108. Shigoka M, Tsuchida A, Matsudo T, et al. Deregulation of miR-92a expression is implicated in hepatocellular carcinoma development. Pathol Int. 2010;60(5):351-357. 
109. Pan Y, Liang H, Liu H, et al. Platelet-secreted microRNA-223 promotes endothelial cell apoptosis induced by advanced glycation end products via targeting the insulin-like growth factor 1 receptor. J Immunol. 2014;192(1):437-446.

110. Cocucci E, Racchetti G, Meldolesi J. Shedding microvesicles: artefacts no more. Trends Cell Biol. 2009;19(2):43-51.

111. Chen X, Liang H, Zhang J, Zen K, Zhang CY. Secreted microRNAs: a new form of intercellular communication. Trends Cell Biol. 2012;22(3):125-132.

112. Vickers KC, Palmisano BT, Shoucri BM, Shamburek RD, Remaley AT. MicroRNAs are transported in plasma and delivered to recipient cells by high-density lipoproteins. Nat Cell Biol. 2011;13(4):423-433.

113. Arroyo JD, Chevillet JR, Kroh EM, et al. Argonaute 2 complexes carry a population of circulating microRNAs independent of vesicles in human plasma. Proc Natl Acad Sci U S A. 2011;108(12):5003-5008.
114. Chen X, Hu ZB, Wang WJ, et al. Identification of ten serum microRNAs from a genome-wide serum microRNA expression profile as novel noninvasive biomarkers for nonsmall cell lung cancer diagnosis. Int J Cancer. 2012;130(7):1620-1628.

115. Chen YY, Li LM, Zhou ZX, Wang N, Zhang CY, Zen K. A pilot study of serum microRNA signatures as a novel biomarker for occult hepatitis B virus infection. Med Microbiol Immun. 2012;201(3):389-395.

116. Yang $\mathrm{CH}$, Wang $\mathrm{C}$, Chen $\mathrm{X}$, et al. Identification of seven serum microRNAs from a genome-wide serum microRNA expression profile as potential noninvasive biomarkers for malignant astrocytomas. Int $J$ Cancer. 2013;132(1):116-127.

117. Luo Y, Wang C, Chen X, et al. Increased serum and urinary microRNAs in children with idiopathic nephrotic syndrome. Clin Chem. 2013;59(4):658-666.

\section{Publish your work in this journal}

The Journal of Hepatocellular Carcinoma is an international, peerreviewed, open access journal that offers a platform for the dissemination and study of clinical, translational and basic research findings in this rapidly developing field. Development in areas including, but not limited to, epidemiology, vaccination, hepatitis therapy, pathology and

\section{Dovepress}

molecular tumor classification and prognostication are all considered for publication. The manuscript management system is completely online and includes a very quick and fair peer-review system, which is all easy to use. Visit http://www.dovepress.com/testimonialsphp to read real quotes from published authors.

Submit your manuscript here: http://www.dovepress.com/journal-of-hepatocellular-carcinoma-journal 